\title{
Construção de um aplicativo móvel para Profilaxia Pós-Exposição ao HIV
}

\author{
Building a mobile application for HIV Post-Exposure Prophylaxis \\ Elaboración de una aplicación móvil para la profilaxis post-exposición al VIH \\ Alexis Pereira da Silva ${ }^{1}$ in https://orcid.org//0000-0002-0770-3089 \\ Barbara Jacqueline Peres Barbosa ${ }^{2}$ @ https://orid.orgy/0000-0003-1509-7207 \\ Robinson Fernandes Camargo ${ }^{3}$ (D) https://orcid.org/0000-0003-0543-453X \\ Lucia Yasuko Izumi Nichiata ${ }^{1}$ ie https://orcid.org/0000-0001-6515-4404
}

Como citar:

Silva AP, Barbosa BJ, Camargo RF, Nichiata LY. Construção de um aplicativo móvel para Profilaxia Pós-Exposição ao HIV. Acta Paul Enferm. 2021;34:eAPE000345.

DOI

http://dx.doi.org/10.37689/actaape/2021A0000345

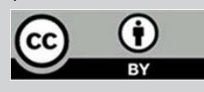

Descritores

Aplicativos móveis; Profilaxia pós-exposição; HIV; Desenvolvimento tecnológico

Keywords

Mobile applications; Post-exposure prophylaxis; HIV; Technological development

Descriptores

Aplicaciones móviles; Profilaxis posterior a la exposición; VIH; Desarrollo tecnológico

Submetido

22 de Fevereiro de 2020

Aceito

9 de Dezembro de 2020

Autor correspondente

Alexis Pereira da Silva

E-mail: alexisps@usp.br

\section{Resumo}

Objetivo: Descrever o processo de construção de um aplicativo móvel para o apoio à Profilaxia Pós-Exposição ao HIV e analisar como os profissionais de saúde respondem aos atributos de sua usabilidade.

Métodos: Estudo de produção tecnológica, construído em quatro fases, entre 2016 e 2018: composição do conteúdo central do app; reunião de um conjunto de ferramentas para o protótipo; testagem do protótipo; teste dos atributos de usabilidade por meio do método empírico. 0 aplicativo PEPtec foi construído baseado nas diretrizes nacional e internacional para profilaxia pós-exposição ao HIV. As respostas dos profissionais de saúde acerca dos atributos de usabilidade (facilidade, utilidade, interface e uso do app) foram analisadas por meio de um questionário semiestruturado aplicado em 28 profissionais dos serviços de atendimento às pessoas com Infecções Sexualmente Transmissíveis de uma Coordenadoria de Saúde do município de São Paulo (SP), Brasil.

Resultados: 0 aplicativo foi disponibilizado para os sistemas operacionais Android e iOS. Foi personalizado para o celular do profissional de saúde para apoio na tomada de decisão na indicação da Profilaxia PósExposição ao HIV. Sobre a interface do aplicativo, os profissionais consideraram que as cores e os ícones eram agradáveis e de fácil identificação. Na análise da usabilidade, os profissionais relataram facilidade no seu uso, ganharam habilidades e conseguiram navegar por todas as telas.

Conclusão: 0 aplicativo móvel responde aos interesses desses profissionais no desfecho da indicação à Profilaxia Pós-Exposição ao HIV por meio desta ferramenta tecnológica.

\section{Abstract}

Objective: To describe the process of building a mobile application to support HIV Post-Exposure Prophylaxis and analyze how healthcare professionals respond to the attributes of its usability.

Methods: A technological production study, built in four phases, between 2016 and 2018: composition of the app's central content; gathering a set of tools for the prototype; prototype testing; testing usability attributes using the empirical method. The PEPtec application was built based on national and international guidelines for post-exposure prophylaxis for HIV. Healthcare professionals' responses about usability attributes (ease, utility, interface and use of the app) were analyzed using a semi-structured questionnaire applied to 28 professionals from the services for people with Sexually Transmitted Infections of a Health Coordination of the municipality of São Paulo (SP), Brazil.

Results: The application was made available for Android and iOS. It was personalized for healthcare professionals' cell phones to support decision-making in the indication of HIV Post-Exposure Prophylaxis. Regarding the application interface, the professionals considered that the colors and icons were pleasant and easy to identify. In the usability analysis, professionals reported ease of use, gained skills and were able to navigate through all screens. 
Conclusion: The mobile application responds to the interests of these professionals in the outcome of indication for Post-Exposure HIV Prophylaxis through this technological tool.

\section{Resumen}

Objetivo: Describir el proceso de elaboración de una aplicación móvil para el apoyo a la profilaxis post-exposición al VIH y analizar cómo responden los profesionales de la salud a los atributos de usabilidad.

Métodos: Estudio de producción tecnológica, elaborado en cuatro fases entre 2016 y 2018: composición del contenido central de la app, reunión del conjunto de herramientas para el prototipo, prueba del prototipo, prueba de los atributos de usabilidad mediante el método empírico. La aplicación PEPtec fue elaborada con base en las directrices nacionales e internacionales para la profilaxis post-exposición al VIH. Las respuestas de los profesionales de la salud acerca de los atributos de usabilidad (facilidad, utilidad, interfaz y uso de la app) fueron analizadas mediante un cuestionario semiestructurado aplicado a 28 profesionales de los servicios de atención a personas con Infecciones de Transmisión Sexual de una Coordinación de Salud del municipio de São Paulo, estado de São Paulo, Brasil.

Resultados: La aplicación fue ofrecida para los sistemas operativos Android e iOS. Se personalizó para los celulares de los profesionales de la salud para apoyar la toma de decisiones sobre la indicación de la profilaxis post-exposición al VIH. Respecto a la interfaz de la aplicación, Ios profesionales consideraron que los colores e íconos eran agradables y de fácil identificación. En el análisis de usabilidad, los profesionales relataron la facilidad de uso, adquirieron habilidades y pudieron navegar por todas las pantallas.

Conclusión: La aplicación móvil responde a los intereses de estos profesionales en los eventos de indicación de profilaxis post-exposición al VIH mediante esta herramienta tecnológica.

\section{Introdução}

A Profilaxia Pós-Exposição (PEP) é uma medida de prevenção contra o Vírus da Imunodeficiência Humana (HIV) e pode reduzir a taxa de nova infecção após a exposição. Consiste no uso diário, durante 28 dias, de doses oral de antirretrovirais, indicados para serem iniciados, preferencialmente em 2 horas e, no máximo, até 72 horas após a exposição ao vírus. Deve ser considerado como atendimento de urgência. ${ }^{(1,2)}$

No Brasil, essa prática vem sendo indicada desde 2010. O relatório de monitoramento clínico do HIV mostra que, no país, houve aumento nas dispensaçóes de antirretrovirais para PEP ao longo dos anos, passando de 17.630, em 2010, para 87.251, em 2017, e para 107.345, em 2018. ${ }^{(3)}$ Infere-se, a partir desses dados, que a população está tendo acesso ao conhecimento sobre a importância de se buscarem os serviços de saúde após possível exposição ao HIV.

Um apoio que tem sido desenvolvido para promover a ampliação da estratégia de prevenção advém dos aplicativos móveis $(a p p)$ construídos para a área da saúde. ${ }^{(4)}$ Definem-se como programas de computador ou softwares instalados em dispositivos eletrônicos móveis, como os celulares Android ou iPhone (iOS), com multiplicidade de funçôes. ${ }^{(5)}$

$\mathrm{O}$ uso adequado dessa tecnologia pode levar a inovadoras formas de melhoria para saúde, e seu potencial é reconhecido pela Organização Mundial da Saúde (OMS). ${ }^{(6)}$ Este órgão reconhece que o uso de app é uma estratégia complementar para o fortalecimento dos cuidados de saúde, ressaltando, porém, a importância da avaliação e do monitoramento desses recursos. ${ }^{(6)}$ Os downloads dos app voltados para essa área são significativos. Dados da Food and Drug Administration, dos Estados Unidos, previa até 2018 que $50 \%$ dos americanos teriam feito download em seus smartphones, incluindo os profissionais de saúde. ${ }^{(7)}$

Estudos indicam a necessidade de análise dos apps quanto à sua usabilidade, ou seja, que sejam testados antes de serem disponibilizados nas lojas virtuais. Devem ser construídos para ter seu uso facilitado para os profissionais da saúde que realizam uma tarefa específica e importante. ${ }^{(8-10)}$ Os desenvolvedores têm repensado o quesito qualidade dos conteúdos centrais dos apps, indicando que devem ser testados na fase de protótipo ou nas versóes iniciais. Pesquisas de usabilidade têm ganhado destaque na produção do conhecimento sobre o uso de apps. ${ }^{(8-12)}$

Os objetivos do presente estudo foram descrever o processo de construçáo de um aplicativo móvel para o apoio a PEP e analisar como os profissionais de saúde respondem aos atributos de sua usabilidade.

\section{Métodos}

Trata-se de um estudo de produção tecnológica destinada à construção de um app para o apoio a PEP. 
É parte do projeto "Protótipo de Dispositivo móvel para o apoio a PEP ao HIV", que obteve aprovação do Comitê de Ética em Pesquisa da Escola de Enfermagem da Universidade de Sáo Paulo (USP) e da Secretaria Municipal de Saúde de São Paulo, conforme Certificado de Apresentação de Apreciação Ética (CAAE) 38350614.3.0000.5392.-

Foi realizado com o apoio da Coordenadoria de Saúde Sudeste do município de São Paulo, ${ }^{(13)}$ participando em todas as quatro fases: composição do conteúdo central do app; reuniáo do conjunto de ferramentas para o protótipo; testagem do protótipo e teste dos atributos de usabilidade do $a p p$.

$\mathrm{Na}$ primeira fase, realizada entre os anos de 2015 e 2016, foram reunidos os conteúdos contidos de diretrizes municipal, nacional e internacional sobre a PEP. A segunda fase, realizada entre 2016 a 2017, deu-se com a participação da equipe da empresa Poli Júnior da Escola Politécnica da USP, a qual desenhou o protótipo. Foi utilizada a ferramenta Ionic Framework, que permite desenvolver um app híbrido para dispositivos móveis, recorrendo à linguagem Angular JS, que simplifica seu desenvolvimento e ajuda a produzir um $a p p$ com visual agradável, o qual utiliza conhecimentos sobre HyperText Markup Language (HTML), Cascading Style Sheet (CSS) e Javascript. ${ }^{(14)} \mathrm{Na}$ terceira fase, realizou-se uma oficina com gestores e profissionais da saúde, da qual participaram seis profissionais da saúde (dois enfermeiros, um médico infectologista e interlocutor do programa de Doenças Sexualmente Transmissíveis e HIV/AIDS da Secretaria Municipal de Saúde, um assistente social, um psicólogo e um dentista), com a finalidade de apresentar e testar o protótipo do app. $\mathrm{O}$ grupo teve por responsabilidade analisar o conteúdo sobre a PEP e a necessidade de adequação da interface do app, no sentido de ser amigável à navegação. Como resultado, houve indicação de inclusão de conteúdos sobre os efeitos adversos possíveis na administração dos antirretrovirais. Não houve sugestôes de adequação do desenho do app e formas de navegação. Na quarta fase, participaram dois pesquisadores que, de forma independente, realizaram entrevistas com os profissionais de serviços de saúde nos meses de dezembro de 2017 a fevereiro de 2018. Os pesquisadores foram aos lo- cais de trabalho, apresentaram o protótipo aos profissionais de saúde e o segmento para a efetuação da testagem para analisar a usabilidade. Nessa fase, 28 profissionais de saúde que atuavam nas unidades da rede especializada dos serviços de atendimento às pessoas com Infecçóes Sexualmente Transmissíveis participaram das entrevistas, como amostragem de conveniência.

$\mathrm{Na}$ análise dos atributos de usabilidade, utilizou-se a recomendação da Norma Brasileira da Associação Brasileira de Normas Técnicas (ABNT) e da International Organization for Standardization (ISO)/International Electrotechnical Commission (IEC) 25062:2011, (15) que sugere a constituição de uma amostra mínima de oito participantes na etapa da testagem desses atributos. Foram inclusos profissionais de saúde de nível superior que aceitaram participar do estudo e assinaram o Termo de Consentimento Livre Esclarecido. Foram excluídos os profissionais de saúde de nível médio e do serviço administrativo. Este critério tem por base os protocolos que demandam profissionais de nível superior com competência para a indicação de PEP, em particular enfermeiros. ${ }^{(16)}$

O questionário da entrevista foi estruturado, dividido em três momentos, utilizando-se, por inspiração, o estudo de Valentim. ${ }^{(17)}$ Fez-se a análise da usabilidade dos apps mensurando os princípios sobre a eficiência percebida pelo usuário, além da percepção das funçóes acessíveis, de forma simples e intuitiva, principalmente por haver pretensão de atingir um conteúdo de extrema importância. Foram analisados nos atributos: facilidade, utilidade, interface e o uso do app na profissão.

No primeiro momento, os participantes preencheram o questionário sobre a experiência com a ferramenta tecnológica em saúde, o conhecimento acerca da utilização de apps móveis em saúde e a frequência no uso de material de apoio para tomada de decisão. Na sequência, foi perguntado se conheciam o PEPtec. Diante de resposta negativa, foi explicado e apresentado o app, convidando-o a fazer o download. No segundo momento, o profissional consultou o dispositivo para responder às perguntas sobre a vivência a respeito da indicação de PEP. As respostas dessa interação foram registradas pelo 
pesquisador, identificando se o profissional teve sucesso (fácil) concluindo sem problemas a tarefa na primeira tentativa; sucesso (difícil) concluindo a tarefa, porém com dificuldades ou insucesso, caso não tenha conseguido completar a tarefa ou desistiu. No último momento, o profissional respondeu ao questionário a respeito dos atributos de usabilidade. Utilizou-se escala do tipo Likert, contendo as opçóes de resposta: concordo totalmente, concordo amplamente, concordo parcialmente, discordo totalmente, discordo amplamente e discordo totalmente. O profissional foi orientado a assinalar uma única resposta. Os três momentos dessa análise foram armazenados e processados em planilhas Microsoft Excel $^{\oplus} 2013$.

Optou-se por apresentar os resultados do teste de usabilidade, agregando as opçóes de resposta "concordo" e "discordo", dado que a distribuição nas variantes mostrou muita dispersão, dificultando a análise.

\section{Resultados}

O PEPtec foi personalizado para o celular dos profissionais de saúde. A primeira versão foi disponibilizada para download, gratuitamente, desde 23 de março de 2016, nos sistemas operacionais Android e iOS. Uma segunda versão foi implementada em 2018 para atualização, tendo como base a publicação do Protocolo Clínico de Diretrizes para PEP. O app auxiliou na avaliação dos riscos a que os usuários estavam expostos ao HIV, de forma a apoiar a tomada de decisão dos profissionais de saúde sobre as recomendaçóes da PEP. A figura 1 exibe a página inicial do app.

Ao selecionar "Home", uma breve explicação sobre o que era a PEP, sua história e a informação sobre sua indicação, bem como comentários gerais sobre a importância da adesão, podiam ser acessados.

A opção "Recomendações PEP para adultos" era o principal foco do app. Foi criada a partir de um algoritmo de decisão, tendo por base a escolha de respostas pelo profissional de saúde em consulta com o usuário sobre as possíveis exposiçóes ao HIV, a qual o direcionava para as seguintes opçôes:

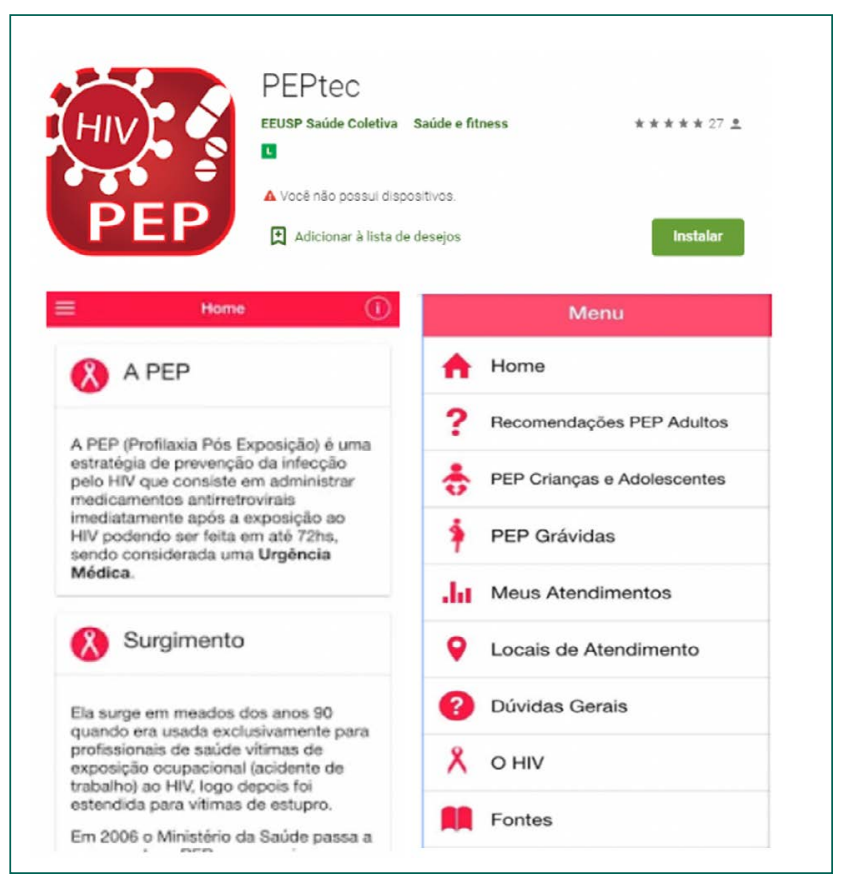

Figura 1. Página inicial do app PEPtec

sexo consensual desprotegido; acidente ocupacional e violência sexual. A opção "Tempo decorrido desde a exposiçáo" - menor ou maior do que 72 horas -, levava a questóes subsequentes: violência sexual, acidente ocupacional e violência sexual. A depender do tempo de exposiçáo, era indicada a PEP.

O ícone "PEP criança e adolescente" apresentava as indicaçôes das medicaçóes para uma possível infecção pelo HIV e informaçóes sobre outras infecçôes. A opção "PEP grávidas” apresentava um alerta sobre algumas contraindicaçóes do uso de antirretrovirais durante a gravidez e a interrupção da amamentação. Havia ainda o recurso "Meus atendimentos" que fornecia uma estatística dos atendimentos. $\mathrm{O}$ app consolidava esses atendimentos em porcentagem, para que o profissional visualizasse que perfil de pacientes que procuraram a PEP. Ele, porém, não armazenava informação pessoal dos pacientes, e o profissional podia removê-la no ícone "Reset estatística".

Ainda nessa tela, era possível visualizar os "Locais de atendimento" com o recurso de Google Map, que indicava a localização em que o usuário se encontrava e os serviços de atendimento a PEP no município de Sáo Paulo (SP). Nos outros municípios e estados do Brasil, redirecionava-se para o link do Centro de Referência e Treinamento DST/ 
AIDS. Havia, ainda, um ícone indicando "Dúvidas gerais", que abordava soluçôes para possíveis dúvidas sobre a PEP. No ícone "O HIV", existia uma descrição sobre a infecção. $\mathrm{O}$ ícone "Fontes" apresentava as principais bibliografias.

Dos 28 profissionais que participaram da análise da usabilidade do $a p p$, a maioria era do sexo feminino $(78,6 \%)$, predominantemente enfermeiros $(53,5 \%)$, possuía experiências com tecnologia $(28,5 \%)$ e o usava com frequência em todos os dias da semana $(25,0 \%)$. Sobre o uso de app relacionados à saúde, mais da metade $(60,7 \%)$ acessava material disponibilizado na internet como apoio à tomada de decisão $(53,5 \%)$, conforme observado na tabela 1 .

Tabela 1. Distribuição dos profissionais de saúde, de acordo com as características sociodemográficas e experiência em uso de tecnologias

\begin{tabular}{|c|c|}
\hline Característica & $\mathrm{n}(\%)$ \\
\hline \multicolumn{2}{|l|}{ Sexo } \\
\hline Feminino & $22(78,6)$ \\
\hline Masculino & $6(21,4)$ \\
\hline \multicolumn{2}{|l|}{ Formação profissional } \\
\hline Enfermeiro & $15(53,5)$ \\
\hline Farmacêutico & $4(14,1)$ \\
\hline Assistente social & $3(10,7)$ \\
\hline Médico & $2(7,1)$ \\
\hline Terapeuta ocupacional & $2(7,1)$ \\
\hline Fonoaudiólogo & $1(3,5)$ \\
\hline Dentista & $1(3,5)$ \\
\hline \multicolumn{2}{|l|}{ Experiência com ferramenta tecnológica em saúde } \\
\hline Não utilizo apps & $4(14,2)$ \\
\hline Não utilizo apps, acesso pelo computador & $4(14,2)$ \\
\hline Já utilizei alguns apps relacionados à saúde & $8(28,5)$ \\
\hline Utilizo apps, em média, dois e três dias por semana & $5(17,8)$ \\
\hline Utilizo apps smartphones todos os dias da semana & $7(25,0)$ \\
\hline \multicolumn{2}{|l|}{ Conhecimento sobre utilização de apps móveis } \\
\hline Nunca utilizei apps desse tipo & $1(3,5)$ \\
\hline Tenho conhecimento desse tipo de aplicação, porém nunca utilizei & $3(10,7)$ \\
\hline Tenho conhecimento desse tipo de aplicação e já utilizei & $7(25,0)$ \\
\hline Utilizo apps desse tipo em tablets e smartphones & $17(60,7)$ \\
\hline \multicolumn{2}{|l|}{ Uso de materiais de apoio para tomada de decisão } \\
\hline Não utilizo & $1(3,5)$ \\
\hline Utilizo apenas o material impresso na unidade & $3(10,7)$ \\
\hline Utilizo material complementar, disponibilizado por meio da internet & $15(53,5)$ \\
\hline Utilizo material complementar a todo momento & $9(32,1)$ \\
\hline
\end{tabular}

Na avaliação do sucesso na indicação da PEP por meio do PEPtec, a maioria dos profissionais obteve sucesso nas etapas (fácil). Todos conseguiram acessar o app e fazer pesquisa direcionada para o perfil violência sexual em menos de 72 horas (100\%). As maiores dificuldades surgiram quando foi necessária a busca dos serviços de saúde, devido à indisponibilidade de conexão de internet, de acordo com a tabela 2 .

Tabela 2. Passos percorridos pelos profissionais de saúde ao navegarem no app móvel

\begin{tabular}{|c|c|c|c|}
\hline \multirow[t]{2}{*}{ Passos de navegação pelo app } & \multicolumn{3}{|c|}{$\begin{array}{l}\text { Avaliação quanto ao sucesso } \\
\text { na indicação de Profilaxia Pós- } \\
\text { Exposição ao HIV por meio do app } \\
\text { PEPtec }\end{array}$} \\
\hline & $\begin{array}{l}\text { Fácil } \\
\mathrm{n}(\%)\end{array}$ & $\begin{array}{l}\text { Difícil } \\
\mathrm{n}(\%)\end{array}$ & $\begin{array}{c}\text { Insucesso } \\
\mathrm{n}(\%)\end{array}$ \\
\hline Acessar 0 app & $28(100)$ & - & - \\
\hline $\begin{array}{l}\text { Pesquisa com o perfil sexo consensual desprotegido } \\
\text { em menos de } 72 \text { horas }\end{array}$ & $22(78,6)$ & $6(21,4)$ & - \\
\hline $\begin{array}{l}\text { Pesquisa com o perfil sexo consensual desprotegido } \\
\text { mais de } 72 \text { horas }\end{array}$ & $27(96,4)$ & $1(3,5)$ & - \\
\hline $\begin{array}{l}\text { Pesquisa com o perfil acidente ocupacional mais } \\
\text { de } 72 \text { horas }\end{array}$ & $26(92,8)$ & $2(7,1)$ & - \\
\hline $\begin{array}{l}\text { Pesquisa com o perfil violência sexual menos de } \\
72 \text { horas }\end{array}$ & $28(100)$ & - & - \\
\hline $\begin{array}{l}\text { Pesquisa: dúvidas sobre efeitos colaterais dos } \\
\text { antirretrovirais: tenofovir e lamivudina }\end{array}$ & $26(92,8)$ & $2(7,1)$ & - \\
\hline $\begin{array}{l}\text { Pesquisa com o perfil sexo consensual desprotegido } \\
\text { menos de } 72 \text { horas, teste rápido negativo, sorologia } \\
\text { negativa do parceiro e não faz parte do grupo de } \\
\text { risco acrescido do HIV }\end{array}$ & $23(82,1)$ & $5(17,8)$ & - \\
\hline $\begin{array}{l}\text { Pesquisa: número de unidades que oferecem a PEP } \\
\text { na regional Sudeste do município de São Paulo }\end{array}$ & $21(95,4)$ & $1(4,7)$ & - \\
\hline $\begin{array}{l}\text { Pesquisa: serviços oferecidos da PEP no município } \\
\text { de Adamantina (SP) }\end{array}$ & $12(57,1)$ & $2(9,5)$ & $7(33,3)$ \\
\hline $\begin{array}{l}\text { Pesquisa: serviços oferecidos da PEP no município } \\
\text { de Umuarama (SP) }\end{array}$ & $5(23,8)$ & $6(28,5)$ & $10(47,6)$ \\
\hline
\end{tabular}

Sobre os atributos de usabilidade do app dos profissionais analisados no estudo, não houve respostas muito diferentes na classificação da escala Likert e, por isso, optou-se pela decisão das opçóes mais expressivas (concordo e discordo). Todos concordaram a respeito da facilidade de uso do PEPtec. Nos atributos utilidade, interface e uso na prática profissional, a maioria dos profissionais concordou que o app é fácil de usar, é útil, tem uma interface amigável e apoia a prática dos profissionais, tendo mais de $90 \%$ de respostas positivas para o uso do app, conforme tabela 3 .

\section{Discussão}

A chegada dos smartphones permitiram que os celulares ultrapassassem o tradicional uso para ligaçôes telefônicas. Atualmente, são considerados um computador de bolso, por trazerem para as máos 
Tabela 3. Atributos de usabilidade do app PEPtec

\begin{tabular}{|c|c|c|}
\hline \multirow[b]{2}{*}{ Atributos de usabilidade do app PEPtec } & \multicolumn{2}{|c|}{ Concordância } \\
\hline & $\begin{array}{c}\text { Concordo } \\
\mathrm{n}(\%)\end{array}$ & $\begin{array}{l}\text { Discordo } \\
\mathrm{n}(\%)\end{array}$ \\
\hline \multicolumn{3}{|l|}{ Facilidade de uso do app } \\
\hline Foi fácil aprender a utilizá-lo & $28(100)$ & \\
\hline Entendia o que acontecia durante o uso do app & $28(100)$ & \\
\hline $\begin{array}{l}\text { Foi fácil ganhar habilidade de uso durante a execução das } \\
\text { atividades no app }\end{array}$ & $28(100)$ & \\
\hline Foi fácil lembrar como utilizá-lo & $28(100)$ & \\
\hline Considero o app fácil de utilizar & $28(100)$ & \\
\hline Consigo navegar bem por todas as telas do app & $28(100)$ & \\
\hline \multicolumn{3}{|l|}{ Utilidade do app } \\
\hline App útil para melhorar minha prática assistencial & $26(92,8)$ & \\
\hline 0 app facilitou a realização das minhas atividades & $2(7,1)$ & \\
\hline \multicolumn{3}{|l|}{ Interface do app } \\
\hline Considero as cores e ícones do app agradáveis & $28(100)$ & \\
\hline $\begin{array}{l}\text { Consigo visualizar bem todos os botões e informações } \\
\text { dentro do app }\end{array}$ & $28(100)$ & \\
\hline $\begin{array}{l}\text { Entendo com facilidade as palavras, nomenclaturas e } \\
\text { ícones do app }\end{array}$ & $28(100)$ & \\
\hline As imagens e ícones no app são de fácil reconhecimento & $27(96,4)$ & $1(3,5)$ \\
\hline Consigo visualizar todas as funcionalidades do $a p p$ & $26(92,8)$ & $2(7,1)$ \\
\hline Consigo navegar bem por todas as telas do app & $27(96,4)$ & $1(3,5)$ \\
\hline \multicolumn{3}{|l|}{ Uso do app na prática profissional } \\
\hline Julgo que o app facilita minha prática assistencial & $26(92,8)$ & $2(7,1)$ \\
\hline Com o app, consigo explorar muito mais sobre a PEP & $27(96,4)$ & $1(3,5)$ \\
\hline $\begin{array}{l}\text { Com o app, tenho maior domínio sobre as práticas } \\
\text { relacionadas ao PEP }\end{array}$ & $26(92,8)$ & $2(7,1)$ \\
\hline $\begin{array}{l}\text { Com o app, tenho mais segurança para realizar a avaliação } \\
\text { da PEP }\end{array}$ & $26(92,8)$ & $2(7,1)$ \\
\hline
\end{tabular}

dos usuários comodidades, em qualquer horário. Dentre as diversas funçóes, pode-se destacar a possibilidade de esses aparelhos oferecerem download de apps, que vão desde entretenimento a suporte de apoio ao profissional de saúde.

No desenvolvimento do protótipo PEPtec, uma das fases foi a análise dos atributos de usabilidade do app. A primeira análise demonstrou que os enfermeiros foram os profissionais que mais responderam à pesquisa.

Há estudos brasileiros ${ }^{(9,10)}$ que abordam apps específicos para esse profissional. Um exemplo é o Oncoaudit, ${ }^{(9)}$ elaborado para auxiliar na consulta de quimioterápicos, como ferramenta de auditoria em enfermagem de contas hospitalares. Ele foi avaliado por enfermeiros auditores seguindo o questionário System Usability Scale, evidenciando que o app segue as necessidades dos usuários. Profissionais de informática também o avaliaram seguindo o método das heurísticas de Nielsen para os quesitos de usabilidade, ao quais sugestóes foram implementadas. ${ }^{(9)}$
O app Cuidar $\operatorname{Tech}^{(10)}$ foi desenvolvido para auxiliar o enfermeiro na avaliação e classificação de risco dos pés de pessoas com diabetes mellitus. Sua usabilidade foi avaliada pela equipe de desenvolvimento seguindo as heurísticas de Nielsen, sendo posteriormente validado por enfermeiros como funcional, confiável, adequado e eficiente. ${ }^{(10)}$

$\mathrm{O}$ WHO Algorithm ${ }^{(18)}$ foi produzido para auxiliar o enfermeiro na avaliação do nível de desidratação em crianças e adultos. O enfermeiro se mostra um profissional que muito utiliza apps de apoio ao seu trabalho. ${ }^{(8-11)}$

O PEPtec foi construído para auxiliar na avaliação dos riscos em que os usuários estejam expostos ao HIV para o apoio na tomada de decisão com base nos apontamentos sobre a PEP. Pode ser utilizado por qualquer profissional que trabalha em pronto-socorro, ambulatório de especialidade, Unidade Básica de Saúde, hospital ou maternidade. No entanto, é importante enfatizar que é permitido ao enfermeiro, segundo a Lei do Exercício Profissional de Enfermagem, integrante da equipe de saúde, prescrever os medicamentos previstos nos protocolos, desde que essa conduta esteja estabelecida em protocolos, normas e rotinas da instituição, e o profissional se sinta, tecnicamente, apto para sua execução. Esse é um profissional estratégico na perspectiva da ampliação da indicação da PEP. ${ }^{(17)}$

No estudo, foi possível perceber que 71,3\% dos profissionais possuem experiências com ferramenta tecnológica na área profissional, e 85,6\% utilizam recursos disponibilizados na internet como apoio na tomada de decisão. Essa vivência com a era tecnológica pode gerar um conhecimento sobre a utilização de apps, tornando esses profissionais mais seletivos e criteriosos nas plataformas virtuais, sobretudo aqueles que agregam conhecimento à profissão.

Nos passos de navegaçáo no PEPtec, identificou-se que alguns dos profissionais tiveram dificuldades quando era necessário indicar uma unidade de saúde fora do município de São Paulo, pois o profissional foi direcionado a um site, fora do $a p p$, e necessitou de acesso à internet. Essa dificuldade pode estar associada ao fato de as unidades de saúde não possuírem acesso à internet sem fios ou o profissional não dispunha de dados móveis em seu celular no momento da análise. 
$\mathrm{O}$ acesso à internet nas instituiçóes de saúde brasileiras é de $91 \%$, sendo $83 \%$ na esfera pública e $99 \%$ na rede privada. Dessas, $98 \%$ das unidades estão situadas nas capitais e $89 \%$ no interior. Contudo, há discrepância quanto à qualidade da conexão à internet. ${ }^{(19)}$

A maioria dos profissionais teve facilidade no manuseio do app, porque, após download, seu uso off-line foi possível. $\mathrm{O}$ envolvimento desses profissionais com o uso do app permite obter dados relevantes de forma eficaz e segura. Dessa maneira, podem ser sugeridas soluçóes para minimizarem as dificuldades vivenciadas durante sua prática profissional, ${ }^{(20)}$ corroborando a iniciativa de ferramentas como o PEPtec no auxílio à tomada de decisão.

$\mathrm{Na}$ análise dos atributos de usabilidade do PEPtec, os profissionais relataram facilidade em seu uso. Estudo de avaliação do app PedsGuide ${ }^{(20)}$ desenvolvido para o apoio na tomada de decisáo do cuidar em pediatria foi similar, mostrando a importância de um app de fácil uso e com funções integradas no cuidar. Outra semelhança foi no estudo de validação do app $\mathrm{BPH},{ }^{(21)}$ construído para apoio na decisão de cirurgia de próstata, ao ser mencionada a relevância de uma ferramenta tecnológica de fácil uso e com opçáo de calculadora de probabilidade que auxilia na indicaçáo cirúrgica.

Sobre a interface do PEPtec, os profissionais consideraram que as cores e os ícones eram agradáveis, conseguiram visualizar todas as informaçóes no app e reconheceram que as imagens e os ícones eram de fácil identificação. No estudo que avaliou a experiência de usabilidade de um aplicativo móvel, ${ }^{(18)}$ houve diferenças das respostas encontradas em comparação com o presente estudo, pois, durante a avaliação dos atributos de usabilidade que analisaram o app Swarm, notaram-se dificuldades enfrentadas pelos usuários em reconhecer ou visualizar ícones no dispositivo, resultando na desistência deles para realizarem algumas atividades. As dificuldades enfrentadas foram direcionadas ao desenvolvedor da tecnologia, para realização de melhorias, que ressaltaram que tais discordâncias não se relacionam com a visibilidade técnica da resolução do produto analisado. ${ }^{(18)}$ Em nenhum momento, houve desistência dos profissionais deste estudo, mas duas melhorias no PEPtec foram indicadas: uma para atualizaçóes das medicaçôes sobre a PEP e a outra para adicionar um gerador de QR-code, que permitirá acessar um novo app que está em fase de desenvolvimento.

Quanto à utilidade do $a p p$, a maior parte dos profissionais respondeu que é eficaz e pode melhorar a prática profissional. A literatura já demonstrou que o uso de app apoia a prática profissional: o Malnutrition Management ${ }^{(22,23)}$ auxilia na gestão do controle da desnutrição em crianças menores de e anos, e o MicroGuide ajuda na navegação das informações sobre os antibióticos. ${ }^{(24)}$ No entanto, no presente estudo, alguns dos profissionais náo concordam com o uso desse recurso tecnológico em sua prática profissional.

O PEPtec não tem a intenção de substituir o conhecimento técnico-científico do profissional de saúde. O app é uma estratégia para o apoio ao profissional na tomada de decisão, em particular o enfermeiro, diante de pacientes expostos ao HIV. Pode contribuir com a melhoria das práticas desse profissional e dos demais membros das equipes para o enfretamento da epidemia do HIV/AIDS e colaborar para a redução da transmissão dessa infecção. A análise sobre a usabilidade é uma condição para o aprimoramento dos apps.

$\mathrm{O}$ estudo apresentou como limitação o longo período de construção e de análise dos atributos de usabilidade do app. Apps móveis podem tornar-se obsoletos rapidamente, à medida que novos conhecimentos são criados e novas tecnologias são desenvolvidas, exigindo um esforço de atualização e aprimoramento constante. Apesar dessa limitação, os resultados da presente pesquisa podem trazer novas contribuiçôes para o estudo desta temática.

\section{Conclusão}

A construção do aplicativo PEPtec foi realizada em quatro fases e representa uma inovação tecnológica desenvolvida no Brasil por ser o primeiro dispositivo móvel de apoio ao profissional de saúde na tomada de decisáo durante a indicação a PEP. A análise das respostas sobre os atributos de usabilidade da primeira versáo do PEPtec demonstrou que há facilidade, utilidade, interface e uso na prática profissional, e o aplicativo responde, portanto, aos interesses no desfecho da indicação da PEP. 


\section{Agradecimentos}

\section{Ao Conselho Nacional de Desenvolvimento Científico e Tecnológico - CNPq pelo financiamen- to desse estudo (Projeto Universal - Processo No. 424852/2016-0).}

\section{Colaborações}

\section{Silva APS, Barbosa BJP, Camargo RF e Nichiata LYI declaram que contribuíram com a concepção do es- tudo, análise e interpretação dos dados, redação do artigo, visão crítica relevante do conteúdo intelec- tual e aprovação da versão final a ser publicada.}

\section{Referências}

1. Word Health Organization (WHO). World Health Organization Guidelines on postexposure prophylaxis for HIV: recommendations for a public health approach. Geneva: WHO; 2015.

2. Brasil. Ministério da Saúde. Departamento de condições Crônicas e Infecções Sexualmente Transmissíveis. Protocolo Clínico e Diretrizes Terapêuticas para Profilaxia Pós-Exposição (PEP) de risco à infecção pelo HIV, IST e Hepatites Virais. Brasília (DF): Ministério da Saúde; 2018.

3. Brasil. Ministério da Saúde. Departamento de condições Crônicas e Infecções Sexualmente Transmissíveis. Relatório de monitoramento clínico do HIV 2019. Brasília (DF): Ministério da Saúde; 2019.

4. Tibes CM, Dias JD, Mascarenhas SH. Aplicativos móveis desenvolvidos para a área da saúde no Brasil: revisão integrativa da literatura. Rev Min Enferm. 2014;18(2):471-8.

5. Centro Latino-americano e do Caribe. Biblioteca Virtual em Saúde. Descritores em ciências da saúde. [citado 2020 Dez 8]. Disponível em: http://decs2016.bvsalud.org/cgi-bin/wxis1660.exe/decsserver/

6. World Health Organization (WHO). mHealth: New horizons for health through mobile technologies. Geneva: WHO; 2011.

7. Department of Health and Human Services Food and Drug Administration (FDA). Transforming FDA's Approach to Digital Health. FDA; 2018 [cited 2019 Sep 10]. Available from: https://www.fda.gov/ news-events/speeches-fda-officials/transforming-fdas-approachdigital-health-04262018

8. Barbosa BJ, Silva AP, Mota TJ, Nichiata LY. Análise do conteúdo central dos aplicativos sobre HIV para smartphones. J Health Inf. 2019;11(1):13-20.

9. Grossi LM, Pisa IT, Marin HF. Oncoaudit: desenvolvimento e avaliação de aplicativos para enfermeiros auditores. Acta Paul Enferm. 2014;27(2):179-85.

10. Vêscovi SJ, Primo CC, Sant'Anna HC, Bringuete ME, Rohr RV, Prado TN, et al. Aplicativo móvel para avaliação dos pés de pessoas com diabetes mellitus. Acta Paul Enferm. 2017;30(6):607-13.
11. Morland LA, Niehaus J, Taft C, Marx BP, Menez U, Mackintosh MA. Using a mobile application in the management of anger problems among veterans: a pilot study. Mil Med. 2016;181(9):990-5.

12. Narváez S, Tobar AM, López DM, Blobel B. Human-centered design of an $\mathrm{m}$-health app for the prevention of burnout syndrome. Stud Health Technol Inform. 2017;228(4):215-9.

13. Prefeitura do Município de São Paulo. São Paulo lança aplicativo sobre PEP para profissionais de saúde. São Paulo: Prefeitura do Município de São Paulo; 2016

14. Ionicframework. Estrutura iônica. [cited 2020 0ct 16]. Available from: https://ionicframework.com/docs

15. Associação Brasileira de Normas Técnicas (ABNT). ABNT NBR ISO/ IEC 25062:2011. Engenharia de Software - Requisitos e avaliação de qualidade de produto de software (SQuaRe) - Formato comum da Indústria (FCl) para relatórios de teste de usabilidade. Brasília (DF): ABNT; 2011.

16. Conselho Regional de Enfermagem (COREN). Câmara Técnica Parecer COREN-SP 033/2019. Ementa: Prescrição de medicamentos por abordagem sindrômica para Prevenção Pré-exposição (PrEP), Prevenção Pós-Exposição (PEP) e Infecções Sexualmente Transmissíveis (ISTs) pelo enfermeiro. São Paulo: COREN; 2019

17. Valentim NM, Rabelo J, Silva W, Coutinho W, Mota A, Conte T. Avaliando a qualidade de um app web móvel através de um teste de usabilidade: um relato de experiência. In: XVIII Simpósio Brasileiro de Qualidade de Software. 2014. [citado 2020 Out 16]. Disponível em: https://www. researchgate.net/publication/276276294_Avaliando_a_Experiencia_ do_Usuario_e_a_Usabilidade_de_um_Aplicativo_Web_Movel_um_ relato_de_experiencia

18. Bilal S, Nelson E, Meisner L, Alam M, Amin SA, Ashenafi Y, et al. Evaluation of standard and mobile health-supported clinical diagnostic tools for assessing dehydration in patients with diarrhea in rural Bangladesh. Am J Trop Med Hyg. 2018;99(1):171-9.

19. Comitê Gestor da Internet no Brasil (CGIB). TIC Saúde. Pesquisa sobre 0 uso das tecnologias de informação e comunicação nos estabelecimentos de saúde brasileiros 2018. $6^{\text {a }}$ ed rev. São Paulo: CGIB; 2018 [citado 2020 Out 16]. Disponível em: https://cetic.br/ media/docs/publicacoes/2/15303120191017-tic_saude_2018_ livro_eletronico.pdf

20. MCCulloh RJ, Fouquet SD, Herigon J, Biondi EA, Kennedy B, Kerns $E$, et al. Development and implementation of a mobile device-based pediatric electronic decision support tool as part of a national practice standardization project. J Am Med Inform Assoc. 2018;25(9):1175-82.

21. Choo MS, Seong JJ, Cho SY, Yoo C, Jeong CW, Ku JH, et al. Development of decision support formulas for the prediction of bladder outlet obstruction and prostatic surgery in patients with lower urinary tract symptom/benign prostatic hyperplasia: part i, development of the formula and its internal validation. Int Neurourol J. 2017;21(Suppl 1):S55-65.

22. Nyumbeka D, Wesson. Using mobile computing to support malnutrition management in South Africa. Proceedings of the Southern African Institute for Computer Scientist and Information Technologists Annual Conference; 2014 [cited 2020 0ct 16]. p. 325-60. Available from: https://www.semanticscholar.org/paper/Using-Mobile-Computing-toSupport-Malnutrition-in-Nyumbeka-Wesson/53332ce81f171b1745e 8201708f628b69ef92b59

24. Panesar P, Jones A, Aldous A, Kranzer K, Halpin E, Fifer H, et al. Attitudes and behaviours to antimicrobial prescribing following introduction of a smartphone App. Plos One. 2016;11(4):1-9. 